\title{
Transformation technique, fixed point theorem and positive solutions for second-order impulsive differential equations with deviating arguments
}

Xuemei Zhang ${ }^{1^{*}}$ and Meiqiang Feng ${ }^{2}$

Correspondence: zxm74@sina.com 'Department of Mathematics and Physics, North China Electric Power University, Beijing, 102206, Republic of China

Full list of author information is available at the end of the article

\section{Springer}

\begin{abstract}
This paper investigates the boundary value problems of second-order impulsive differential equations with deviating arguments

$$
\left\{\begin{array}{l}
x^{\prime \prime}(t)+\omega(t) f(t, x(\alpha(t)))=0, \quad t \in J, t \neq t_{k,} \\
x\left(t_{k}^{+}\right)-x\left(t_{k}\right)=c_{k} x\left(t_{k}\right), \quad k=1,2, \ldots, n, \\
a x(0)-b x^{\prime}(0)=a x(1)-b x^{\prime}(1)=\int_{0}^{1} h(s) x(t) d t,
\end{array}\right.
$$

where $\left\{c_{k}\right\}$ is a real sequence with $c_{k}>-1, k=1,2, \ldots, n, \omega$ may be singular at $t=0$ and/or $t=1$. Several new and more general results are obtained for the existence of positive solutions for the above problem by using transformation technique and Krasnosel'skii's fixed point theorem. We discuss our problems under two cases when the deviating arguments are delayed and advanced. The approach to deal with the impulsive term is different from earlier approaches. It is the first paper where the transformation technique and a fixed point theorem for cones are applied to second-order differential equations with impulsive effects and deviating arguments. An example is included to verify the theoretical results.
\end{abstract}

Keywords: advanced and delayed arguments; impulsive differential equations; transformation technique; fixed point theorem; positive solutions

\section{Introduction}

Impulsive differential equations, which provide a natural description of observed evolution processes, are regarded as important mathematical tools for better understanding of several real world problems in applied sciences, such as population dynamics, ecology, biological systems, biotechnology, industrial robotic, pharmacokinetics, optimal control, etc. Therefore, the study of this class of impulsive differential equations has gained prominence and it is a rapidly growing field. For the general theory of impulsive differential equations, we refer the reader to [1-3], whereas the applications of impulsive differential equations can be found in [4-14]. Nieto and O'Regan [15] pointed out that in a secondorder differential equation $u^{\prime \prime}=f\left(t, u, u^{\prime}\right)$ one usually considers impulses in the position $u$ and the velocity $u^{\prime}$. However, in the motion of spacecraft one has to consider instantaneous impulses depending on the position that result in jump discontinuities in velocity,

@2014 Zhang and Feng; licensee Springer. This is an Open Access article distributed under the terms of the Creative Commons Attribution License (http://creativecommons.org/licenses/by/2.0), which permits unrestricted use, distribution, and reproduction in any medium, provided the original work is properly cited. 
but with no change in position [16]. The impulses only on velocity occur also in impulsive mechanics [17].

Some classical tools such as bifurcation theory $[18,19]$, fixed point theorems in cones [20-24], the method of lower and upper solutions $[25,26]$, the theory of critical point theory and variational methods $[7,15,27-30]$ and the technique via appropriate transformation [31-34] have been widely used to study impulsive differential equations. But it is quite difficult to apply these approaches to an impulsive differential equation with deviating arguments; therefore, there was no result in this area for a long time. Only in the recent eight years, there appeared a few articles which dealt with some impulsive differential equations with deviating arguments by using fixed point theorems in cones [35-38]. Motivated by [35-38], in this article we shall use a different approach to discuss the existence of positive solutions for a class of impulsive differential equations with deviating arguments.

Consider the second-order nonlinear impulsive differential equation of the type

$$
\left\{\begin{array}{l}
x^{\prime \prime}(t)+\omega(t) f(t, x(\alpha(t)))=0, \quad t \in J, t \neq t_{k}, \\
x\left(t_{k}^{+}\right)-x\left(t_{k}\right)=c_{k} x\left(t_{k}\right), \quad k=1,2, \ldots, n, \\
a x(0)-b x^{\prime}(0)=a x(1)-b x^{\prime}(1)=\int_{0}^{1} h(s) x(t) d t,
\end{array}\right.
$$

where $J=[0,1], f \in C\left(J \times R^{+}, R^{+}\right), R^{+}=[0,+\infty), t_{k}(k=1,2, \ldots, n$, here $n$ is a fixed positive integer) are fixed points with $0<t_{1}<t_{2}<\cdots<t_{k}<\cdots<t_{n}<1, a, b>0,\left\{c_{k}\right\}$ is a real sequence with $c_{k}>-1, k=1,2, \ldots, n, x\left(t_{k}^{+}\right)(k=1,2, \ldots, n)$ represent the right-hand limit of $x(t)$ at $t_{k}, h \in C[0,1]$ is nonnegative.

Throughout this paper we assume that $\alpha(t) \not \equiv t$ on $J=[0,1]$. In addition, $\omega, f, c_{k}, \alpha$ and $h$ satisfy

$\left(\mathrm{H}_{1}\right) \omega \in C((0,1),[0,+\infty))$ with $0<\int_{0}^{1} \omega(s) d s<\infty$ and $\omega$ does not vanish on any subinterval of $(0,1)$;

$\left(\mathrm{H}_{2}\right) f \in C([0,1] \times[0,+\infty),[0,+\infty)), \alpha \in C(J, J)$;

$\left(\mathrm{H}_{3}\right)\left\{c_{k}\right\}$ is a real sequence with $c_{k}>-1, k=1,2, \ldots, n, c(t):=\prod_{0<t_{k}<t}\left(1+c_{k}\right)$;

$\left(\mathrm{H}_{4}\right) h \in C[0,1]$ is nonnegative with $v \in[0, a)$, where

$$
v=\int_{0}^{1} h(t) c(t) d t
$$

Remark 1.1 Throughout this paper, we always assume that a product $c(t):=\prod_{0<t_{k}<t}\left(1+c_{k}\right)$ equals unity if the number of factors is equal to zero, and let

$$
c_{M}=\max _{t \in J} c(t), \quad c_{m}=\min _{t \in J} c(t), \quad c^{-1}(t)=\prod_{0<t_{k}<t}\left(1+c_{k}\right)^{-1} .
$$

Remark 1.2 Combining $\left(\mathrm{H}_{3}\right)$ and the definition of $c(t)$, we know that $c(t)$ is a step function and bounded on $J$, and

$$
c(t)>0, \quad \forall t \in J, \quad c(t)=1, \quad \forall t \in\left[0, t_{1}\right] .
$$

Some special cases of (1.1) have been investigated. For example, Zhang and Feng [34] considered problem (1.1) under the case that $\omega(t) \equiv 1$ and $\alpha(t) \equiv t$ on $J$. By using fixed 
point theories in cones, the authors proved the existence of positive solutions for problem (1.1).

At the same time, a class of boundary value problems with delay has been investigated; for example, see [39-44]. It is not difficult to see that the corresponding functions $f$ appearing on the right-hand side depend on $x(t-\tau), \tau>0$, where initial functions $x$ are given on the initial set, for example, $[-\tau, 0]$. Jankowski $[45,46]$ pointed out that in such cases $\alpha(t)=t-\tau$, there are some problems with a constant delay $\tau$. If we consider the differential problem on intervals $[0, k]$, where $k \leq \tau$, then it means that we have no delays; we have such a situation in paper [44]. If $k>\tau$, then it is easy to solve the differential equation on $[0, \tau]$, since we have the solution on the initial set $[-\tau, 0]$. Continuing this process, we can find a solution on the whole interval $[0, k]$ by using the method of steps. In the present paper, for example, the deviating argument $\alpha$ can have a form $\alpha(t)=\rho t=t-(1-\rho) t$ with a fixed number $\rho \in(0,1)$, so the delay $(1-\rho) t$ is a function of $t$. In this case, the initial set reduces to one point $t=0$, and we cannot apply the step method. To the authors' knowledge, it is the first paper when positive solutions have been investigated for a class of second-order impulsive differential equations with deviating arguments both of advanced and delayed type.

Remark 1.3 There are almost no papers, except [35-37], studying second-order impulsive differential equations with deviating arguments using fixed point theory. However, in [35-37], Jankowski only considered $\omega \in C([0,1], \infty)$, not $\omega$ is singular at $t=0$ and/or $t=1$, and dealt with the nonlinear term that is in the form of $f(y(t))$, not $f(t, c(t) y(t))$; see (2.12).

Remark 1.4 Comparing with [35-37], we transform problem (1.1) into a differential system without impulse, i.e., the technique to deal with impulses is completely different from that of [35-37]. According to the authors' knowledge, it is probably the first paper where this technique is applied to second-order impulsive boundary value problems with deviating arguments.

Remark 1.5 The technique to deal with $f(t, c(t) y(t))$ is completely different from that of Zhang et al. [31], Zhang et al. [32] and Sun et al. [33].

Being directly inspired by [31-37], the authors will prove several new and more general results for the existence of positive solutions for problem (1.1) by using fixed point theories.

The organization of this paper is as follows. In Section 2, we present some definitions and lemmas which are needed throughout this paper. In particular, we transform problem (1.1) into a differential system without impulse. In Section 3, we use a fixed point theorem to obtain the existence of positive solutions for problem (1.1) with advanced argument $\alpha$. Finally, in Section 4, we formulate sufficient conditions under which delayed problem (1.1) has positive solutions. In particular, our results in these sections are new when $\alpha(t) \equiv t$ on $t \in J$.

\section{Preliminaries}

In this section, we first present some definitions and lemmas which are needed throughout this paper. 
Definition 2.1 (see [47]) Let $E$ be a real Banach space over $R$. A nonempty closed set $P \subset E$ is said to be a cone provided that

(i) $a u+b v \in P$ for all $u, v \in P$ and all $a \geq 0, b \geq 0$, and

(ii) $u,-u \in P$ implies $u=0$.

Every cone $P \subset E$ induces an ordering in $E$ given by $x \leq y$ if and only if $y-x \in P$.

Definition 2.2 The map $\beta$ is said to be a nonnegative continuous concave functional on a cone $P$ of a real Banach space $E$ provided that $\beta: P \rightarrow[0, \infty)$ is continuous and

$$
\beta(t x+(1-t) y) \geq t \beta(x)+(1-t) \beta(y)
$$

for all $x, y \in P$ and $0 \leq t \leq 1$.

Definition 2.3 A function $x(t)$ is said to be a solution of problem (1.1) on $J$ if:

(i) $x(t)$ is absolutely continuous on each interval $\left(0, t_{1}\right]$ and $\left(t_{k}, t_{k+1}\right], k=1,2, \ldots, n$;

(ii) for any $k=1,2, \ldots, n, x\left(t_{k}^{+}\right), x\left(t_{k}^{-}\right)$exist and $x\left(t_{k}^{-}\right)=x\left(t_{k}\right)$;

(iii) $x(t)$ satisfies (1.1).

We shall reduce problem (1.1) to a system without impulse. To this goal, firstly by means of the transformation

$$
x(t)=c(t) y(t)
$$

we convert problem (1.1) into

$$
\left\{\begin{array}{l}
-y^{\prime \prime}(t)=\omega(t) c^{-1}(t) f(t, c(\alpha(t)) y(\alpha(t))), \quad t \in J, \\
a y(0)-b y^{\prime}(0)=\int_{0}^{1} h(s) c(s) y(s) d s, \\
a c(1) y(1)-b c(1) y^{\prime}(1)=\int_{0}^{1} h(s) c(s) y(s) d s .
\end{array}\right.
$$

The following lemmas will be used in the proof of our main results.

\section{Lemma 2.1 Assume that $\left(\mathrm{H}_{1}\right)-\left(\mathrm{H}_{4}\right)$ hold. Then}

(i) If $y(t)$ is a solution of problem (2.2) on $J$, then $x(t)=c(t) y(t)$ is a solution of problem (1.1) on $\mathrm{J}$;

(ii) If $x(t)$ is a solution of problem (1.1) on $J$, then $y(t)=c^{-1}(t) x(t)$ is a solution of problem (2.2) on J.

Proof The proof is similar to that of Lemma 2.1 in [34].

Lemma 2.2 If $\left(\mathrm{H}_{1}\right)-\left(\mathrm{H}_{4}\right)$ hold, then problem (2.2) has a solution $y$, and $y$ can be expressed in the form

$$
y(t)=\int_{0}^{1} H(t, s) c^{-1}(s) f(t, c(\alpha(s)) y(\alpha(s))) d s,
$$

where

$$
H(t, s)=G(t, s)+\frac{1}{a-v} \int_{0}^{1} G(\tau, s) c(\tau) h(\tau) d \tau,
$$




$$
G(t, s)=\frac{1}{a(a+2 b)} \begin{cases}(b+a t)(a(1-s)+b), & 0 \leq t \leq s \leq 1 \\ (b+a s)(a(1-t)+b), & 0 \leq s \leq t \leq 1\end{cases}
$$

Proof The proof is similar to that of Lemma 2.1 in [48].

Lemma 2.3 Let $\xi \in(0,1), G$ and $H$ be given as in Lemma 2.2. Then we have the following results:

$$
\begin{array}{ll}
G(t, s) \leq G(s, s), \quad H(t, s) \leq H(s, s)=\frac{a}{a-v} G(s, s), \quad \forall t, s \in J, \\
G(t, s) \geq \delta G(s, s), \quad H(t, s) \geq \delta H(s, s)=\frac{\delta a}{a-v} G(s, s), \quad \forall t \in[\xi, 1], s \in J,
\end{array}
$$

where

$$
\delta=\frac{b}{b+a}
$$

Proof Relation (2.6) is simple to prove. Note that

$$
\begin{aligned}
& \frac{G(t, s)}{G(s, s)}=\frac{b+a t}{b+a s} \geq \frac{b+a \xi}{b+a} \quad \text { for } s \leq t, \\
& \frac{G(t, s)}{G(s, s)}=\frac{b+a(1-t)}{b+a(1-s)} \geq \frac{b}{b+a} \quad \text { for } t \leq s,
\end{aligned}
$$

for $t \in[\xi, 1], s \in J$.

Similarly, we can prove that $H(t, s) \geq \delta H(s, s)$ for $t \in[\xi, 1], s \in J$. Hence, it follows from $G(t, s) \geq \delta G(s, s)$ that

$$
H(t, s) \geq \delta H(s, s)=\frac{\delta a}{a-v} G(s, s), \quad \forall t \in[\xi, 1], s \in J
$$

This gives the proof of Lemma 2.3.

Remark 2.1 Noticing that $a, b>0$, it follows from (2.4) and (2.5) that

$$
0<\beta_{1} \leq G(t, s) \leq \beta_{2}, \quad \forall t, s \in J
$$

and

$$
0<\beta^{*} \leq H(t, s) \leq \beta, \quad \forall t, s \in J,
$$

where

$$
\beta_{1}=\frac{b^{2}}{a(a+2 b)}, \quad \beta_{2}=\frac{a+2 b}{4 a}, \quad \beta^{*}=\frac{b^{2}}{a(a+2 b)(a-v)}, \quad \beta=\frac{a+2 b}{4(a-v)}
$$

Let $E=C[0,1]$. Then $E$ is a real Banach space with the norm $\|\cdot\|$ defined by

$$
\|y\|=\max _{t \in J}|y(t)|, \quad y \in E .
$$


Define a cone $K$ in $E$ by

$$
K=\left\{y \in E: y(t) \geq 0, \min _{t \in[\xi, 1]} y(t) \geq \delta\|y\|\right\}
$$

Also, define for $r$ a positive number $\Omega_{r}$ by

$$
\Omega_{r}=\{y \in E:\|y\|<r\}
$$

Note that $\partial \Omega_{r}=\{y \in E:\|y\|=r\}$.

Define $T: K \rightarrow K$ by

$$
(T y)(t)=\int_{0}^{1} H(t, s) \omega(s) c^{-1}(s) f(s, c(\alpha(s)) y(\alpha(s))) d s
$$

Lemma 2.4 Assume that $\left(\mathrm{H}_{1}\right)-\left(\mathrm{H}_{4}\right)$ hold. Then $T(K) \subset K$ and $T: K \rightarrow K$ is completely continuous.

Proof For $y \in K$, it follows from (2.6) and (2.12) that

$$
\begin{aligned}
(T y)(t) & =\int_{0}^{1} H(t, s) \omega(s) c^{-1}(s) f(s, c(\alpha(s)) y(\alpha(s))) d s \\
& \leq \int_{0}^{1} H(s, s) \omega(s) c^{-1}(s) f(s, c(\alpha(s)) y(\alpha(s))) d s, \quad t \in J .
\end{aligned}
$$

It follows from (2.7), (2.12) and (2.13) that

$$
\begin{aligned}
\min _{t \in[\xi, 1]}(T y)(t) & =\min _{t \in[\xi, 1]} \int_{0}^{1} H(t, s) \omega(s) c^{-1}(s) f(s, c(\alpha(s)) y(\alpha(s))) d s \\
& \geq \delta \int_{0}^{1} H(s, s) \omega(s) c^{-1}(s) f(s, c(\alpha(s)) y(\alpha(s))) d s \\
& \geq \delta\|T y\| .
\end{aligned}
$$

Thus, $T(K) \subset K$.

Next, by arguments similar to those of Theorem 1 in [49], one can prove that $T: K \rightarrow K$ is completely continuous. So it is omitted, and the lemma is proved.

Remark 2.2 From (2.12), we know that $y \in E$ is a solution of problem (2.2) if and only if $y$ is a fixed point of the operator $T$.

From Lemma 2.1 and Remark 2.2, we can obtain the following results.

Lemma 2.5 Assume that $\left(\mathrm{H}_{1}\right)-\left(\mathrm{H}_{4}\right)$ hold. Then

(i) If $x(t)$ is a solution of problem (1.1) on $J$, then $y(t)=c^{-1}(t) x(t)$ is a fixed point of $T$;

(ii) If $y(t)$ is a fixed point of $T$, then $x(t)=c(t) y(t)$ is a solution of problem (1.1) on $J$. 
In the rest of this section, we state a well-known fixed point theorem which we need later.

Lemma 2.6 (see [47]) Let $P$ be a cone in a real Banach space E. Assume that $\Omega_{1}, \Omega_{2}$ are bounded open sets in $E$ with $0 \in \Omega_{1}, \bar{\Omega}_{1} \subset \Omega_{2}$. If

$$
A: P \cap\left(\bar{\Omega}_{2} \backslash \Omega_{1}\right) \rightarrow P
$$

is completely continuous such that either

(i) $\|A x\| \leq\|x\|, \forall x \in P \cap \partial \Omega_{1}$ and $\|A x\| \geq\|x\|, \forall x \in P \cap \partial \Omega_{2}$, or

(ii) $\|A x\| \geq\|x\|, \forall x \in P \cap \partial \Omega_{1}$ and $\|A x\| \leq\|x\|, \forall x \in P \cap \partial \Omega_{2}$,

then $A$ has at least one fixed point in $P \cap\left(\bar{\Omega}_{2} \backslash \Omega_{1}\right)$.

\section{Existence of positive solutions for problem (1.1) under $\alpha(t) \geq t$ on $J$}

For convenience, we introduce the following notations:

$$
\begin{array}{ll}
f^{0}=\limsup _{y \rightarrow 0} \max _{t \in J} \frac{f(t, y)}{y}, & f_{0}=\liminf _{y \rightarrow 0} \min _{t \in J} \frac{f(t, y)}{y}, \\
f^{\infty}=\limsup _{y \rightarrow \infty} \max _{t \in J} \frac{f(t, y)}{y}, & f_{\infty}=\liminf _{y \rightarrow \infty} \min _{t \in J} \frac{f(t, y)}{y} .
\end{array}
$$

We also define as [50] $i_{0}=$ number of zeros in the set $\left\{f^{0}, f^{\infty}\right\}$ and $i_{\infty}=$ number of infinities in the set $\left\{f_{0}, f_{\infty}\right\}$. Sun and Li [51] pointed out that $i_{0}, i_{\infty}=0,1$ or 2 , and there are six possible cases: (i) $i_{0}=0$ and $i_{\infty}=0$; (ii) $i_{0}=0$ and $i_{\infty}=1$; (iii) $i_{0}=0$ and $i_{\infty}=2$; (iv) $i_{0}=1$ and $i_{\infty}=0$; (v) $i_{0}=1$ and $i_{\infty}=1$; and (vi) $i_{0}=2$ and $i_{\infty}=0$. By using Krasnosel'skii's fixed point theorem in a cone, some results are obtained for the existence of at least one or two positive solutions of problem (1.1) for $\alpha(t) \geq t$ on $J$ under the above six possible cases.

\subsection{For the case $\alpha(t) \geq t$ on $J$ under $i_{0}=1$ and $i_{\infty}=1$}

In this subsection, we discuss the existence of a single positive solution for problem (1.1) for $\alpha(t) \geq t$ on $J$ under $i_{0}=1$ and $i_{\infty}=1$.

For convenience, we introduce the following notations:

$$
\gamma=\int_{0}^{1} \omega(s) d s, \quad \gamma_{1}=\int_{\xi}^{1} \omega(s) d s
$$

Theorem 3.1 Assume that $\left(\mathrm{H}_{1}\right)-\left(\mathrm{H}_{4}\right)$ hold. If $i_{0}=1$ and $i_{\infty}=1$, then problem (1.1) has at least one positive solution.

Proof First, we consider the case $f^{0}=0$ and $f_{\infty}=\infty$. Since $f^{0}=0$, then there exists $r_{1}>0$ such that

$$
f(t, y) \leq \frac{c_{m}}{\beta \gamma c_{M}} y \quad\left(\forall t \in J, 0 \leq y \leq r_{1}\right) .
$$

Since $0 \leq t \leq \alpha(t) \leq 1$ on $J$, it follows from $0 \leq y(t) \leq r_{1}$ on $J$ that

$$
0 \leq y(\alpha(t)) \leq r_{1} \quad \text { for } t \in J
$$


Let $r=\min \left\{r_{1}, \frac{1}{c_{M}} r_{1}\right\}$. Then, for $y \in K \cap \partial \Omega_{r}$, we have $0 \leq y(t) \leq r \leq r_{1}$ for $t \in J$, and then

$$
c(\alpha(t)) y(\alpha(t)) \leq c_{M}\|y\|=c_{M} r \leq r_{1}, \quad t \in J
$$

Consequently, for any $t \in J$ and $y \in K \cap \partial \Omega_{r}$, (2.9) and (2.12) imply

$$
\begin{aligned}
(T y)(t) & =\int_{0}^{1} H(t, s) \omega(s) c^{-1}(s) f(s, c(\alpha(s)) y(\alpha(s))) d s \\
& \leq \beta c_{m}^{-1} \int_{0}^{1} \omega(s) \frac{c_{m}}{\gamma \beta c_{M}} c(\alpha(s)) y(\alpha(s)) d s \\
& \leq \beta c_{m}^{-1} \frac{c_{m}}{\gamma \beta c_{M}} c_{M} \int_{0}^{1} \omega(s) y(\alpha(s)) d s \\
& \leq \frac{1}{\gamma} \int_{0}^{1} \omega(s) d s\|y\| \\
& =\|y\|,
\end{aligned}
$$

which implies

$$
\|T y\| \leq\|y\|, \quad \forall y \in K \cap \partial \Omega_{r} .
$$

Next turning to $f_{\infty}=\infty$, there exists $\hat{r}$ satisfying $0<r_{1}<\hat{r}$ such that

$$
f(t, y) \geq \frac{c_{M}}{c_{m} \delta \beta^{*} \gamma_{1}} y, \quad \forall t \in[\xi, 1], y \geq \hat{r}
$$

Since $\xi \leq t \leq \alpha(t) \leq 1$ on $J$, it follows from $y(t) \geq \hat{r}$ on $[\xi, 1]$ that

$$
y(\alpha(t)) \geq \hat{r} \quad \text { for } t \in[\xi, 1]
$$

Let $R>\max \left\{\hat{r}, \frac{\hat{r}}{\delta c_{m}}\right\}$. Then, for $y \in K \cap \partial \Omega_{R}$, we have

$$
c(\alpha(t)) y(\alpha(t)) \geq c_{m} y(\alpha(t)) \geq c_{m} \delta\|y\| \geq \hat{r}, \quad t \in[\xi, 1] .
$$

Hence, for $y \in K \cap \partial \Omega_{R}$, it follows from (2.9) and (2.12) that

$$
\begin{aligned}
(T y)(t) & =\int_{0}^{1} H(t, s) \omega(s) c^{-1}(s) f(s, c(\alpha(s)) y(\alpha(s))) d s \\
& \geq \beta^{*} \int_{0}^{1} \omega(s) c^{-1}(s) f(s, c(\alpha(s)) y(\alpha(s))) d s \\
& \geq \beta^{*} c_{M}^{-1} \int_{\xi}^{1} \omega(s) \frac{c_{M}}{c_{m} \delta \beta^{*} \gamma_{1}} c(\alpha(s)) y(\alpha(s)) d s \\
& \geq \beta^{*} c_{M}^{-1} \frac{c_{M}}{c_{m} \delta \beta^{*} \gamma_{1}} c_{m} \int_{\xi}^{1} \omega(s) y(\alpha(s)) d s \\
& \geq \frac{1}{\delta \gamma_{1}} \int_{\xi}^{1} \omega(s) d s \delta\|y\| \\
& =\|y\|
\end{aligned}
$$


which implies

$$
\|T y\| \geq\|y\|, \quad \forall y \in K \cap \partial \Omega_{R} .
$$

Thus by (i) of Lemma 2.6, it follows that $T$ has a fixed point $y$ in $K \cap\left(\bar{\Omega}_{R} \backslash \Omega_{r}\right)$ with

$$
r \leq\|y\| \leq R
$$

Lemma 2.5 implies that problem (1.1) has at least one positive solution $x$ with

$$
c_{m} r \leq\|x\| \leq c_{M} R
$$

This gives the proof of Theorem 3.1.

Remark 3.1 For $i_{0}=1$ and $i_{\infty}=1$, there is another case $f^{\infty}=0$ and $f_{0}=\infty$. However, at the moment, we give no information on the existence of a positive solution for problem (1.1) if we change $f^{0}=0$ and $f_{\infty}=\infty$ into $f^{\infty}=0$ and $f_{0}=\infty$ in Theorem 3.1.

\subsection{For the case $\alpha(t) \geq t$ on $J$ under $i_{0}=0$ and $i_{\infty}=0$}

In this subsection, we discuss the existence for the positive solutions of problem (1.1) under $i_{0}=0$ and $i_{\infty}=0$. For convenience, we introduce the following notations:

$$
f_{0}^{\rho}=\max \left\{\max _{t \in J} \frac{f(t, y)}{\rho}: y \in[0, \rho]\right\}
$$

and

$$
l=\frac{c_{m}}{\beta \gamma}, \quad L=\frac{c_{M}}{c_{m} \beta^{*} \delta \gamma_{1}} .
$$

Now, we shall state and prove the following main result.

Theorem 3.2 Suppose that $\left(\mathrm{H}_{1}\right)-\left(\mathrm{H}_{4}\right)$ hold and $\alpha(t) \geq t$ on J. In addition, let the following two conditions hold:

$\left(\mathrm{H}_{5}\right)$ There exist $l>0$ and $\rho_{1}>0$ such that $f_{0}^{\rho_{1}} \leq l$;

$\left(\mathrm{H}_{6}\right)$ There exist $\eta>0$ and $\rho_{2}>0$ such that $f(t, y) \geq \eta$ for $t \in J, y \geq \rho_{2}$; furthermore, $\rho_{1} \neq \rho_{2}$.

Then problem (1.1) has at least one positive solution.

Proof Without loss of generality, we may assume that $\rho_{1}<\rho_{2}$. Considering $f_{0}^{\rho_{1}} \leq l$, we have $f(t, y) \leq l \rho_{1}$ for $0 \leq y \leq \rho_{1}, t \in J$.

Since $0 \leq t \leq \alpha(t) \leq 1$ on $J$, it follows from $0 \leq y(t) \leq \rho_{1}$ on $J$ that

$$
0 \leq y(\alpha(t)) \leq \rho_{1}
$$

Let $\rho=\min \left\{\rho_{1}, \frac{1}{c_{M}} \rho_{1}\right\}$. Then, for $y \in K \cap \partial \Omega_{\rho}$, we have $0 \leq y(t) \leq \rho \leq \rho_{1}$ for $t \in J$, and then

$$
c(\alpha(t)) y(\alpha(t)) \leq c_{M}\|y\|=c_{M} \rho \leq \rho_{1}, \quad t \in J .
$$


Consequently, for any $t \in J$ and $y \in K \cap \partial \Omega_{\rho}$, (2.9) and (2.12) imply

$$
\begin{aligned}
(T y)(t) & =\int_{0}^{1} H(t, s) \omega(s) c^{-1}(s) f(s, c(\alpha(s)) y(\alpha(s))) d s \\
& \leq \beta c_{m}^{-1} l \rho_{1} \int_{0}^{1} \omega(s) d s \\
& =\beta c_{m}^{-1} l \rho_{1} \gamma \\
& =\rho_{1},
\end{aligned}
$$

which implies

$$
\|T y\| \leq\|y\|, \quad \forall y \in K \cap \partial \Omega_{\rho} .
$$

On the other hand, from $\left(\mathrm{H}_{6}\right)$, when $\rho_{2}$ is fixed, there exists $\eta>0$ such that

$$
f(t, y) \geq \eta \geq \max \left\{\rho_{2}, \frac{\rho_{2}}{\delta c_{m}}\right\} \times \frac{c_{M}}{\beta^{*} \gamma_{1}}
$$

for $t \in J$ and $y \geq \rho_{2}$. Since $0 \leq t \leq \alpha(t) \leq 1$ on $J$, it follows from $y(t) \geq \rho_{2}$ on $J$ that

$$
y(\alpha(t)) \geq \rho_{2}
$$

Let $\bar{\rho}=\max \left\{\rho_{2}, \frac{\rho_{2}}{\delta c_{m}}\right\}$. Then, for $y \in K \cap \partial \Omega_{\bar{\rho}}$, we have

$$
c(t) y(t) \geq c_{m} y(t) \geq c_{m} \delta\|y\| \geq \rho_{2}, \quad t \in[\xi, 1] .
$$

Hence, for $y \in K \cap \partial \Omega_{\bar{\rho}}$, it follows from (2.9) and (2.12) that

$$
\begin{aligned}
(T y)(t) & =\int_{0}^{1} H(t, s) \omega(s) c^{-1}(s) f(s, c(\alpha(s)) y(\alpha(s))) d s \\
& \geq \beta^{*} \int_{0}^{1} \omega(s) c^{-1}(s) f(s, c(\alpha(s)) y(\alpha(s))) d s \\
& \geq \beta^{*} c_{M}^{-1} \int_{\xi}^{1} \omega(s) \eta d s \\
& \geq \beta^{*} c_{M}^{-1} \eta \int_{\xi}^{1} \omega(s) d s \\
& =\beta^{*} c_{M}^{-1} \eta \gamma_{1} \\
& \geq \beta^{*} c_{M}^{-1} \gamma_{1} \bar{\rho} \frac{c_{M}}{\beta^{*} \gamma_{1}} \\
& =\bar{\rho},
\end{aligned}
$$

which implies

$$
\|T y\| \geq\|y\|, \quad \forall y \in K \cap \partial \Omega_{\bar{\rho}} .
$$


Thus by (i) of Lemma 2.6, it follows that $T$ has a fixed point $y$ in $K \cap\left(\bar{\Omega}_{\rho_{2}} \backslash \Omega_{\rho_{1}}\right)$ with

$$
\rho \leq\|y\| \leq \bar{\rho} .
$$

Thus, it follows from Lemma 2.5 that problem (1.1) has at least one positive solution $x$ with

$$
c_{m} \rho \leq\|x\| \leq c_{M} \bar{\rho}
$$

This finishes the proof of Theorem 3.2.

We remark that condition $\left(\mathrm{H}_{5}\right)$ in Theorem 3.2 can be replaced by the following condition:

$\left(\mathrm{H}_{5}\right)^{\prime} f^{0} \leq l$

which is a special case of $\left(\mathrm{H}_{5}\right)$.

Corollary 3.1 Suppose that $\left(\mathrm{H}_{1}\right)-\left(\mathrm{H}_{4}\right),\left(\mathrm{H}_{5}\right)^{\prime},\left(\mathrm{H}_{6}\right)$ hold and $\alpha(t) \geq t$ on J. Then problem (1.1) has at least one positive solution.

Proof We show that $\left(\mathrm{H}_{5}\right)^{\prime}$ implies $\left(\mathrm{H}_{5}\right)$. Suppose that $\left(\mathrm{H}_{5}\right)^{\prime}$ holds. Then there exists a positive number $\rho_{1} \neq \rho_{2}$ such that

$$
\frac{f(t, y)}{y} \leq l, \quad t \in J, 0<y \leq \rho_{1} .
$$

Hence, we obtain

$$
f(t, y) \leq l y \leq l \rho_{1}, \quad t \in J, 0<y \leq \rho_{1} .
$$

Therefore, $\left(\mathrm{H}_{5}\right)$ holds. Hence, by Theorem 3.2, problem (1.1) has at least one positive solution.

Theorem 3.3 Suppose that $\left(\mathrm{H}_{1}\right)-\left(\mathrm{H}_{5}\right)$ hold and $\alpha(t) \geq t$ on J. In addition, let the following condition hold:

$\left(\mathrm{H}_{7}\right) f_{\infty} \geq L$

Then problem (1.1) has at least one positive solution.

Proof The proof is similar to those of (3.2) and (3.3), respectively.

Corollary 3.2 Suppose that $\left(\mathrm{H}_{1}\right)-\left(\mathrm{H}_{4}\right),\left(\mathrm{H}_{5}\right)^{\prime},\left(\mathrm{H}_{7}\right)$ hold and $\alpha(t) \geq t$ on J. Then problem (1.1) has at least one positive solution.

3.3 For the case $\alpha(t) \geq t$ on $J$ under $i_{0}=1$ and $i_{\infty}=0$ or $i_{0}=0$ and $i_{\infty}=1$ In this subsection, we discuss the existence for the positive solutions of problem (1.1) for the case $\alpha(t) \geq t$ on $J$ under $i_{0}=1$ and $i_{\infty}=0$ or $i_{0}=0$ and $i_{\infty}=1$. 
Theorem 3.4 Suppose that $\left(\mathrm{H}_{1}\right)-\left(\mathrm{H}_{4}\right)$ hold, $\alpha(t) \geq t$ on $J$ and $f^{0} \in[0, l)$ and $f_{\infty} \in(L, \infty)$. Then problem (1.1) has at least one positive solution.

Proof The proof is similar to that of Theorem 3.2.

Theorem 3.5 Suppose that $\left(\mathrm{H}_{1}\right)-\left(\mathrm{H}_{4}\right)$ hold, $\alpha(t) \geq t$ on $J$ and $f_{0} \in(L, \infty)$ and $f^{\infty} \in[0, l)$. Then problem (1.1) has at least one positive solution.

Proof Consider $f_{0} \in(L, \infty)$, then there exists $\rho_{1}>0$ such that $f(t, y)>L y$ for $0 \leq y \leq \rho_{1}$, $t \in J$.

Since $0 \leq t \leq \alpha(t) \leq 1$ on $J$, it follows from $0 \leq y(t) \leq \rho_{1}$ on $J$ that

$$
0 \leq y(\alpha(t)) \leq \rho_{1}
$$

Let $\rho=\min \left\{\rho_{1}, \frac{1}{c_{M}} \rho_{1}\right\}$. Then, for $y \in K \cap \partial \Omega_{\rho}$, we have $0 \leq y(t) \leq \rho \leq \rho_{1}$ for $t \in J$, and then

$$
c(\alpha(t)) y(\alpha(t)) \leq c_{M}\|y\|=c_{M} \rho \leq \rho_{1}, \quad t \in J .
$$

Consequently, for $y \in K \cap \partial \Omega_{\rho}$, it follows from (2.9) and (2.12) that

$$
\begin{aligned}
(T y)(t) & =\int_{0}^{1} H(t, s) \omega(s) c^{-1}(s) f(s, c(\alpha(s)) y(\alpha(s))) d s \\
& \geq \beta^{*} \int_{0}^{1} \omega(s) c^{-1}(s) f(s, c(\alpha(s)) y(\alpha(s))) d s \\
& \geq \beta^{*} c_{M}^{-1} \int_{\xi}^{1} \omega(s) L c(\alpha(s)) y(\alpha(s)) d s \\
& \geq \beta^{*} c_{M}^{-1} L c_{m} \delta\|y\| \int_{\xi}^{1} \omega(s) d s \\
& =\beta^{*} c_{M}^{-1} L c_{m} \delta\|y\| \gamma_{1} \\
& \geq\|y\|,
\end{aligned}
$$

which implies

$$
\|T y\| \geq\|y\|, \quad \forall y \in K \cap \partial \Omega_{\rho} .
$$

Next, turn to $f^{\infty} \in[0, l)$. In fact, we can show that $f^{\infty} \in[0, l)$ implies $\left(\mathrm{H}_{5}\right)$.

Let $\tau \in\left(f^{\infty}, l\right)$. Then there exists $r>\tau$ such that $\max _{t \in J} f(t, y) \leq \tau y$ for $y \in[r, \infty)$. Let

$$
\beta=\max \left\{\max _{t \in J} f(t, y): 0 \leq y \leq r\right\} \quad \text { and } \quad \rho_{1}^{*}>\max \left\{\frac{\beta}{l-\tau}, \rho, c_{M} \rho\right\} .
$$

Then we have

$$
\max _{0 \leq t \leq 1} f(t, y) \leq \tau y+\beta \leq \tau \rho_{1}^{*}+\beta<l \rho_{1}^{*}, \quad \forall y \in\left[0, \rho_{1}^{*}\right]
$$

This implies that $f_{0}^{\rho_{1}^{*}} \leq l$. Hence, $f^{\infty} \in[0, l)$ implies that $\left(\mathrm{H}_{5}\right)$. 
Similarly to the proof of (3.3), we have

$$
\|T y\| \leq\|y\|, \quad \forall y \in K \cap \partial \Omega_{\rho^{*}}
$$

where $\rho^{*}=\min \left\{\rho_{1}^{*}, \frac{1}{c_{M}} \rho_{1}^{*}\right\}$.

Thus by (ii) of Lemma 2.6, it follows that $T$ has a fixed point $y$ in $K \cap\left(\bar{\Omega}_{\rho^{*}} \backslash \Omega_{\rho}\right)$ with

$$
\rho \leq\|y\| \leq \rho^{*} .
$$

This finishes the proof of Theorem 3.5.

From Theorems 3.4 and 3.5, we have the following result.

Corollary 3.3 Suppose that $f^{0}=0$ and condition $\left(\mathrm{H}_{6}\right)$ in Theorem 3.2 hold. Then problem (1.1) has at least one positive solution.

Theorem 3.6 Suppose that $\left(\mathrm{H}_{1}\right)-\left(\mathrm{H}_{4}\right), \alpha(t) \geq t$ on $J, f^{0} \in(0, l)$ and $f_{\infty}=\infty$. Then problem (1.1) has at least one positive solution.

Proof The proof is similar to that of Theorem 3.2.

Theorem 3.7 Suppose $\left(\mathrm{H}_{1}\right)-\left(\mathrm{H}_{4}\right), \alpha(t) \geq t$ on $J, f_{0}=\infty$ and $f^{\infty} \in(0, l)$. Then problem $(1.1)$ has at least one positive solution.

Proof The proof is similar to that of Theorem 3.2.

From Theorems 3.6 and 3.7, the following corollaries are easily obtained.

Corollary 3.4 Suppose that $f^{0}=\infty$ and condition $\left(\mathrm{H}_{5}\right)$ in Theorem 3.2 hold. Then problem (1.1) has at least one positive solution.

Corollary 3.5 Suppose that $f_{\infty}=\infty$ and condition $\left(\mathrm{H}_{5}\right)$ in Theorem 3.2 hold. Then problem (1.1) has at least one positive solution.

\subsection{For the case $\alpha(t) \geq t$ on $J$ under $i_{0}=0$ and $i_{\infty}=2$ or $i_{0}=2$ and $i_{\infty}=0$}

In this subsection we study the existence of multiple positive solutions for problem (1.1) for the case $\alpha(t) \geq t$ on $J$ under $i_{0}=0$ and $i_{\infty}=2$ or $i_{0}=2$ and $i_{\infty}=0$.

Combining the proofs of Theorems 3.1 and 3.2 , the following theorem is easily proved.

Theorem 3.8 Suppose that $\left(\mathrm{H}_{1}\right)-\left(\mathrm{H}_{4}\right), \alpha(t) \geq t$ on $J, i_{0}=0$ and $i_{\infty}=2$ and condition $\left(\mathrm{H}_{5}\right)$ of Theorem 3.2 hold. Then problem (1.1) has at least two positive solutions.

Corollary 3.6 Suppose that $\left(\mathrm{H}_{1}\right)-\left(\mathrm{H}_{4}\right), \alpha(t) \geq$ ton $J, i_{0}=0$ and $i_{\infty}=2$ and condition $\left(\mathrm{H}_{5}\right)^{\prime}$ of Corollary 3.1 hold. Then problem (1.1) has at least two positive solutions.

Remark 3.2 Noticing Remark 3.1, at the moment we give no information on the existence of a positive solution for problem (1.1) under $i_{0}=2$ and $i_{\infty}=0$. 


\section{Positive solutions of problem (1.1) for the case of $\alpha(t) \leq t$ on $J$}

Now we deal with problem (1.1) for the case of $\alpha(t) \leq t$ on $J$. Similarly as in Lemmas 2.3 and 2.4, we can prove the following results.

Lemma 4.1 Let $\xi \in(0,1), G$ and $H$ be given as in Lemma 2.2. Then we have the following results:

$$
G(t, s) \geq \delta G(s, s), \quad H(t, s) \geq \delta H(s, s)=\frac{\delta a}{a-v} G(s, s), \quad \forall t \in[0, \xi], s \in J,
$$

where

$$
\delta=\frac{b}{b+a} .
$$

Proof Note that

$$
\begin{aligned}
& \frac{G(t, s)}{G(s, s)}=\frac{b+a t}{b+a s} \geq \frac{b}{b+a} \quad \text { for } s \leq t, \\
& \frac{G(t, s)}{G(s, s)}=\frac{b+a(1-t)}{b+a(1-s)} \geq \frac{b+a(1-\xi)}{b+a} \quad \text { for } t \leq s,
\end{aligned}
$$

for $t \in[0, \xi], s \in J$.

Similarly, we can prove that $H(t, s) \geq \delta H(s, s)$ for $t \in[0, \xi], s \in J$. Hence, it follows from $G(t, s) \geq \delta G(s, s)$ that

$$
H(t, s) \geq \delta H(s, s)=\frac{\delta a}{a-v} G(s, s), \quad \forall t \in[0, \xi], s \in J .
$$

This gives the proof of Lemma 4.1.

Let $E$ be as defined in Section 2. We define a cone $K^{*}$ in $E$ by

$$
K^{*}=\left\{u \in E: u \geq 0, \min _{t \in[0, \xi]} u(t) \geq \delta\|u\|\right\} .
$$

It is easy to see that $K^{*}$ is a closed convex cone of $E$.

Define $T^{*}: K^{*} \rightarrow E$ by

$$
\left(T^{*} y\right)(t)=\int_{0}^{1} H(t, s) \omega(s) c^{-1}(s) f(s, c(\alpha(s)) y(\alpha(s))) d s .
$$

It is clear that $y(t)$ is a positive solution of problem (1.1) if and only of $y$ is a fixed point of $T^{*}$.

By analogous methods, we have the following results. Here we only give the proof of Lemma 4.1.

Lemma 4.2 Assume that $\left(\mathrm{H}_{1}\right)-\left(\mathrm{H}_{4}\right)$ hold. Then $T^{*}\left(K^{*}\right) \subset K^{*}$ and $T^{*}: K^{*} \rightarrow K^{*}$ is completely continuous.

Lemma 4.3 Assume that $\left(\mathrm{H}_{1}\right)-\left(\mathrm{H}_{4}\right)$ hold. Then

(i) If $x(t)$ is a solution of problem (1.1) on $J$, then $y(t)=c^{-1}(t) x(t)$ is a fixed point of $T^{*}$;

(ii) If $y(t)$ is a fixed point of $T^{*}$, then $x(t)=c(t) y(t)$ is a solution of problem (1.1) on $J$. 
Similar to the proof of that in Section 3, we have the following results.

4.1 For the case $\alpha(t) \leq t$ on $J$ under $i_{0}=1$ and $i_{\infty}=1$

For convenience, we introduce the following notation:

$$
\gamma_{1}^{*}=\int_{0}^{\xi} \omega(s) d s
$$

Theorem 4.1 Assume that $\left(\mathrm{H}_{1}\right)-\left(\mathrm{H}_{4}\right)$ hold. If $i_{0}=1$ and $i_{\infty}=1$, then problem (1.1) has at least one positive solution.

Proof First, we consider the case $f^{0}=0$ and $f_{\infty}=\infty$. Since $f^{0}=0$, then there exists $r_{1}>0$ such that

$$
f(t, y) \leq \frac{c_{m}}{\beta \gamma c_{M}} y \quad\left(\forall t \in J, 0 \leq y \leq r_{1}\right) .
$$

Since $0 \leq \alpha(t) \leq t \leq 1$ on $J$, it follows from $0 \leq y(t) \leq r_{1}$ on $J$ that

$$
0 \leq y(\alpha(t)) \leq r_{1} \quad \text { for } t \in J
$$

Let $r=\min \left\{r_{1}, \frac{1}{c_{M}} r_{1}\right\}$. Then, for $y \in K^{*} \cap \partial \Omega_{r}$, we have $0 \leq y(t) \leq r \leq r_{1}$ for $t \in J$, and then

$$
c(\alpha(t)) y(\alpha(t)) \leq c_{M}\|y\|=c_{M} r \leq r_{1}, \quad t \in J .
$$

Consequently, for any $t \in J$ and $y \in K^{*} \cap \partial \Omega_{r},(2.9)$ and (2.12) imply

$$
\begin{aligned}
\left(T^{*} y\right)(t) & =\int_{0}^{1} H(t, s) \omega(s) c^{-1}(s) f(s, c(\alpha(s)) y(\alpha(s))) d s \\
& \leq \beta c_{m}^{-1} \int_{0}^{1} \omega(s) \frac{c_{m}}{\gamma \beta c_{M}} c(\alpha(s)) y(\alpha(s)) d s \\
& \leq \beta c_{m}^{-1} \frac{c_{m}}{\gamma \beta c_{M}} c_{M} \int_{0}^{1} \omega(s) y(\alpha(s)) d s \\
& \leq \frac{1}{\gamma} \int_{0}^{1} \omega(s) d s\|y\| \\
& =\|y\|,
\end{aligned}
$$

which implies

$$
\left\|T^{*} y\right\| \leq\|y\|, \quad \forall y \in K^{*} \cap \partial \Omega_{r} .
$$

Next we consider $f_{\infty}=\infty$, there exists $\hat{r}$ satisfying $0<r_{1}<\hat{r}$ such that

$$
f(t, y) \geq \frac{c_{M}}{c_{m} \delta \alpha \gamma_{1}} y, \quad \forall t \in[0, \xi], y \geq \hat{r} .
$$

Since $\alpha(t) \leq t \leq \xi$ on $J$, it follows from $y(t) \geq \hat{r}$ on $[0, \xi]$ that

$$
y(\alpha(t)) \geq \hat{r} \text { for } t \in[0, \xi] .
$$


Let $R>\max \left\{\hat{r}, \frac{\hat{r}}{\delta c_{m}}\right\}$. Then, for $y \in K^{*} \cap \partial \Omega_{R}$, we have

$$
c(\alpha(t)) y(\alpha(t)) \geq c_{m} y(\alpha(t)) \geq c_{m} \delta\|y\| \geq \hat{r}, \quad t \in[0, \xi] .
$$

Hence, for $y \in K^{*} \cap \partial \Omega_{R}$, it follows from (2.9) and (2.12) that

$$
\begin{aligned}
\left(T^{*} y\right)(t) & =\int_{0}^{1} H(t, s) \omega(s) c^{-1}(s) f(s, c(\alpha(s)) y(\alpha(s))) d s \\
& \geq \alpha c_{M}^{-1} \int_{0}^{\xi} \omega(s) \frac{c_{M}}{c_{m} \delta \alpha \gamma_{1}} c(\alpha(s)) y(\alpha(s)) d s \\
& \geq \alpha c_{M}^{-1} \frac{c_{M}}{c_{m} \delta \alpha \gamma_{1}} c_{m} \int_{0}^{\xi} \omega(s) y(\alpha(s)) d s \\
& \geq \frac{1}{\delta \gamma_{1}^{*}} \int_{0}^{\xi} \omega(s) d s \delta\|y\| \\
& =\|y\|
\end{aligned}
$$

which implies

$$
\left\|T^{*} y\right\| \geq\|y\|, \quad \forall y \in K^{*} \cap \partial \Omega_{R}
$$

Thus by (i) of Lemma 2.6, it follows that $T^{*}$ has a fixed point $y$ in $K^{*} \cap\left(\bar{\Omega}_{R} \backslash \Omega_{r}\right)$ with

$$
r \leq y(t) \leq R
$$

This finishes the proof of Theorem 4.1.

Remark 4.1 For $i_{0}=1$ and $i_{\infty}=1$, there is another case $f^{\infty}=0$ and $f_{0}=\infty$. However, at the moment, we give no information on the existence of a positive solution for problem (1.1) if we change $f^{0}=0$ and $f_{\infty}=\infty$ into $f^{\infty}=0$ and $f_{0}=\infty$ in Theorem 4.1.

\subsection{For the case $\alpha(t) \leq t$ on $J$ under $i_{0}=0$ and $i_{\infty}=0$}

In this subsection, we discuss the existence for the positive solutions of problem (1.1) under $i_{0}=0$ and $i_{\infty}=0$. For convenience, we introduce the following notation:

$$
L^{*}=\frac{c_{M}}{c_{m} \alpha \delta \gamma_{1}^{*}} .
$$

Now, we shall state and prove the following main result.

Theorem 4.2 Suppose that $\left(\mathrm{H}_{1}\right)-\left(\mathrm{H}_{5}\right)$ hold and $\alpha(t) \leq t$ on J. In addition, let the following condition hold:

$\left(\mathrm{H}_{6}^{*}\right)$ There exist $\eta^{*}>0$ and $\rho_{2}>0$ such that $f(t, y) \geq \eta^{*}$ for $t \in J, y \geq \rho_{2}$; furthermore, $\rho_{1} \neq \rho_{2}$.

Then problem (1.1) has at least one positive solution. 
Corollary 4.1 Suppose that $\left(\mathrm{H}_{1}\right)-\left(\mathrm{H}_{4}\right),\left(\mathrm{H}_{5}\right)^{\prime},\left(\mathrm{H}_{6}^{*}\right)$ hold and $\alpha(t) \leq t$ on J. Then problem (1.1) has at least one positive solution.

Theorem 4.3 Suppose that $\left(\mathrm{H}_{1}\right)-\left(\mathrm{H}_{5}\right)$ hold and $\alpha(t) \leq t$ on J. In addition, let the following condition hold:

$\left(\mathrm{H}_{7}^{*}\right) f_{\infty} \geq L^{*}$

Then problem (1.1) has at least one positive solution.

Corollary 4.2 Suppose that $\left(\mathrm{H}_{1}\right)-\left(\mathrm{H}_{4}\right),\left(\mathrm{H}_{5}\right)^{\prime},\left(\mathrm{H}_{7}^{*}\right)$ hold and $\alpha(t) \geq t$ on J. Then problem (1.1) has at least one positive solution.

4.3 For the case $\alpha(t) \leq t$ on $J$ under $i_{0}=1$ and $i_{\infty}=0$ or $i_{0}=0$ and $i_{\infty}=1$

Theorem 4.4 Suppose that $\left(\mathrm{H}_{1}\right)-\left(\mathrm{H}_{4}\right)$ hold, $\alpha(t) \leq t$ on $J$ and $f^{0} \in[0, l)$ and $f_{\infty} \in\left(L^{*}, \infty\right)$. Then problem (1.1) has at least one positive solution.

Theorem 4.5 Suppose that $\left(\mathrm{H}_{1}\right)-\left(\mathrm{H}_{4}\right)$ hold, $\alpha(t) \leq t$ on $J$ and $f_{0} \in\left(L^{*}, \infty\right)$ and $f^{\infty} \in[0, l)$. Then problem (1.1) has at least one positive solution.

4.4 For the case $\alpha(t) \leq t$ on $J$ under $i_{0}=0$ and $i_{\infty}=2$ or $i_{0}=2$ and $i_{\infty}=0$

Combining the proofs of Theorems 4.1 and 4.2, the following theorem is easily proved.

Theorem 4.6 Suppose that $\left(\mathrm{H}_{1}\right)-\left(\mathrm{H}_{4}\right), \alpha(t) \leq t$ on $J, i_{0}=0$ and $i_{\infty}=2$ and condition $\left(\mathrm{H}_{5}\right)$ of Theorem 4.2 hold. Then problem (1.1) has at least two positive solutions.

Corollary 4.3 Suppose that $\left(\mathrm{H}_{1}\right)-\left(\mathrm{H}_{4}\right), \alpha(t) \leq t$ on $J, i_{0}=0$ and $i_{\infty}=2$ and condition $\left(\mathrm{H}_{5}\right)^{\prime}$ of Corollary 4.1 hold. Then problem (1.1) has at least two positive solutions.

\section{An example}

To illustrate how our main results can be used in practice, we present an example.

Example 5.1 Consider the following boundary value problem:

$$
\left\{\begin{array}{l}
x^{\prime \prime}(t)+\omega(t) f(t, x(\alpha(t)))=0, \quad t \in J, t \neq \frac{1}{2}, \\
x\left(\frac{1}{2}^{+}\right)-x\left(\frac{1}{2}\right)=\frac{1}{2} x\left(\frac{1}{2}\right), \quad k=1, \\
2 x(0)-x^{\prime}(0)=2 x(1)-x^{\prime}(1)=\int_{0}^{1} x(t) d t
\end{array}\right.
$$

where $\alpha \in C(J, J), \alpha(t) \geq t$ on $J$ and

$$
\omega(t)=\frac{1}{\sqrt{t}}, \quad f(t, x)=\sqrt[n]{1+t^{n}} x^{n}
$$

here $n \geq 2$ is a positive integral number.

This means that problem (5.1) involves the advanced argument $\alpha$. For example, we can take $\alpha(t)=\sqrt[3]{t}$. It is clear that $\omega$ is singular at $t=0$ and $f$ is both nonnegative and continuous. 
Problem (5.1) can be regarded as a problem of the form (1.1), where $a=2, b=1, h(t) \equiv 1$, and

$$
c(t)= \begin{cases}1, & 0 \leq t \leq \frac{1}{2} \\ \frac{3}{2}, & \frac{1}{2}<t \leq 1\end{cases}
$$

Hence

$$
\delta=\frac{1}{3}, \quad v=\int_{0}^{1} h(t) c(t) d t=\frac{5}{4}, \quad c_{M}=\frac{3}{2}, \quad c_{m}=1,
$$

and

$$
\beta^{*}=\frac{b^{2}}{a(a+2 b)(a-v)}=\frac{1}{6}, \quad \beta=\frac{a+2 b}{4(a-v)}=\frac{4}{3} .
$$

It follows from the definition of $\omega, f, c$ and $h$ that $\left(\mathrm{H}_{1}\right)-\left(\mathrm{H}_{4}\right)$ hold, and

$$
f^{0}=0, \quad f_{\infty}=\infty
$$

Hence, by Theorem 3.1, the conclusion follows, and the proof is complete.

\section{Competing interests}

The authors declare that they have no competing interests.

\section{Authors' contributions}

XZ completed the main study and carried out the results of this article. MF checked the proofs and verified the calculation. All the authors read and approved the final manuscript.

\section{Author details}

'Department of Mathematics and Physics, North China Electric Power University, Beijing, 102206, Republic of China. ${ }^{2}$ School of Applied Science, Beijing Information Science \& Technology University, Beijing, 100192, Republic of China.

\section{Acknowledgements}

This work is sponsored by the project NSFC $(11301178,11171032)$, the Fundamental Research Funds for the Central Universities (2014ZZD10, 2014MS58) and the improving project of graduate education of Beijing Information Science and Technology University (YJT201416). The authors are grateful to anonymous referees for their constructive comments and suggestions, which has greatly improved this paper.

\section{Received: 13 November 2014 Accepted: 25 November 2014 Published: 08 Dec 2014}

\section{References}

1. Lakshmikantham, V, Bainov, D, Simeonov, P: Theory of Impulsive Differential Equations. World Scientific, Singapore (1989)

2. Samoilenko, A, Perestyuk, N: Impulsive Differential Equations. World Scientific, Singapore (1995)

3. Benchohra, M, Henderson, J, Ntouyas, S: Impulsive Differential Equations and Inclusions. Hindawi, New York (2006)

4. Ding, W, Han, M: Periodic boundary value problem for the second order impulsive functional differential equations. Appl. Math. Comput. 155, 709-726 (2004)

5. Agarwal, RP, O'Regan, D: Multiple nonnegative solutions for second order impulsive differential equations. Appl. Math. Comput. 114, 51-59 (2000)

6. Nieto, JJ, López, R: Boundary value problems for a class of impulsive functional equations. Comput. Math. Appl. 55, 2715-2731 (2008)

7. Feng, M, Du, B, Ge, W: Impulsive boundary value problems with integral boundary conditions and one-dimensional p-Laplacian. Nonlinear Anal. TMA 70, 3119-3126 (2009)

8. Li, J, Nieo, JJ: Existence of positive solutions for multipoint boundary value problem on the half-line with impulses. Bound. Value Probl. 2009, 834158 (2009)

9. Guo, D: Multiple positive solutions of impulsive nonlinear Fredholm integral equations and applications. J. Math. Anal. Appl. 173, 318-324 (1993)

10. Liu, B, Yu, J: Existence of solution of $m$-point boundary value problems of second-order differential systems with impulses. Appl. Math. Comput. 125, 155-175 (2002) 
11. Lin, $X$, Jiang, D: Multiple positive solutions of Dirichlet boundary value problems for second order impulsive differential equations. J. Math. Anal. Appl. 321, 501-514 (2006)

12. Shen, J, Wang, W: Impulsive boundary value problems with nonlinear boundary conditions. Nonlinear Anal. TMA 69, 4055-4062 (2008)

13. Wei, Z: Periodic boundary value problems for second order impulsive integro-differential equations of mixed type in Banach spaces. J. Math. Anal. Appl. 195, 214-229 (1995)

14. Hristova, S, Bainov, D: Monotone-iterative techniques of V. Lakshmikantham for a boundary value problem for systems of impulsive differential-difference equations. J. Math. Anal. Appl. 197, 1-13 (1996)

15. Nieto, JJ, O'Regan, D: Variational approach to impulsive differential equations. Nonlinear Anal., Real World Appl. 10, 680-690 (2009)

16. Liu, X, Willms, A: Impulsive controllability of linear dynamical systems with applications to maneuvers of spacecraft. Math. Probl. Eng. 2, 277-299 (1996)

17. Pasquero, S: Ideality criterion for unilateral constraints in time-dependent impulsive mechanics. J. Math. Phys. 46 , $112904(2005)$

18. Liu, Y, O'Regan, D: Multiplicity results using bifurcation techniques for a class of boundary value problems of impulsive differential equations. Commun. Nonlinear Sci. Numer. Simul. 16, 1769-1775 (2011)

19. Ma, R, Yang, B, Wang, Z: Positive periodic solutions of first-order delay differential equations with impulses. Appl. Math. Comput. 219, 6074-6083 (2013)

20. Hao, X, Liu, L, Wu, Y: Positive solutions for $n$ th-order singular nonlocal boundary value problems. Bound. Value Probl. $2007,74517(2007)$

21. Liu, L, Liu, Z, Wu, Y: Infinite boundary value problems for $n$ th-order nonlinear impulsive integro-differential equations in Banach spaces. Nonlinear Anal. TMA 67, 2670-2679 (2007)

22. Feng, M: Positive solutions for a second-order $p$-Laplacian boundary value problem with impulsive effects and two parameters. Abstr. Appl. Anal. (2014). doi:10.1155/2014/534787

23. Zhang, X, Feng, M, Ge, W: Existence of solutions of boundary value problems with integral boundary conditions for second-order impulsive integro-differential equations in Banach spaces. J. Comput. Appl. Math. 233, 1915-1926 (2010)

24. Yan, J: Existence of positive periodic solutions of impulsive functional differential equations with two parameters. J. Math. Anal. Appl. 327, 854-868 (2007)

25. Liu, X, Guo, D: Method of upper and lower solutions for second-order impulsive integro-differential equations in a Banach space. Comput. Math. Appl. 38, 213-223 (1999)

26. Guo, D: Extremal solutions for $n$ th-order impulsive integro-differential equations on the half-line in Banach spaces. Nonlinear Anal. TMA 65, 677-696 (2006)

27. Zhou, J, Li, Y: Existence and multiplicity of solutions for some Dirichlet problems with impulsive effects. Nonlinear Anal. TMA 71, 2856-2865 (2009)

28. Xiao, J, Nieto, JJ, Luo, Z: Multiplicity of solutions for nonlinear second order impulsive differential equations with linear derivative dependence via variational methods. Commun. Nonlinear Sci. Numer. Simul. 17, 426-432 (2012)

29. Tian, Y, Ge, W: Applications of variational methods to boundary value problem for impulsive differential equations. Proc. Edinb. Math. Soc. 51, 509-527 (2008)

30. Tian, Y, Ge, W: Variational methods to Sturm-Liouville boundary value problem for impulsive differential equations. Nonlinear Anal. TMA 72, 277-287 (2010)

31. Zhang, $X$, Yang, $X, G e, W$ : Positive solutions of $n$ th-order impulsive boundary value problems with integral boundary conditions in Banach spaces. Nonlinear Anal. TMA 71, 5930-5945 (2009)

32. Zhang, X, Yan, J, Zhao, A: Existence of positive periodic solutions for an impulsive differential equation. Nonlinear Anal. TMA 68, 3209-3216 (2008)

33. Sun, $\mathrm{X}, \mathrm{Huo}, \mathrm{H}, \mathrm{Ma}, \mathrm{C}$ : Periodic solutions of a class of impulsive neutral delay differential equation. Appl. Math. Comput. 219, 3947-3955 (2012)

34. Zhang, $X$, Feng, M: Transformation techniques and fixed point theories to establish the positive solutions of second order impulsive differential equations. J. Comput. Appl. Math. 271, 117-129 (2014)

35. Jankowski, T: Positive solutions of three-point boundary value problems for second order impulsive differential equations with advanced arguments. Appl. Math. Comput. 197, 179-189 (2008)

36. Jankowski, T: Three positive solutions to second order three-point impulsive differential equations with deviating arguments. Int. J. Comput. Math. 87, 215-225 (2010)

37. Jankowski, T: Existence of positive solutions to second order four-point impulsive differential problems with deviating arguments. Comput. Math. Appl. 58, 805-817 (2009)

38. Jankowski, T: Positive solutions to third-order impulsive Sturm-Liouville boundary value problems with deviating arguments and one-dimensional p-Laplacian. Dyn. Syst. Appl. 20, 575-586 (2011)

39. Zhang, X, Feng, M: Green's function and positive solutions for a second-order singular boundary value problem with integral boundary conditions and a delayed argument. Abstr. Appl. Anal. (2014). doi:10.1155/2014/393187

40. Bai, $\mathrm{D}, \mathrm{Xu}, \mathrm{Y}$ : Positive solutions of second-order two-delay differential systems with twin parameter. Nonlinear Anal. TMA 63, 601-617 (2005)

41. Du, B, Hu, X, Ge, W: Positive solutions to a type of multi-point boundary value problem with delay and one-dimensional p-Laplacian. Appl. Math. Comput. 208, 501-510 (2009)

42. Jiang, D: Multiple positive solutions for boundary value problems of second order delay differential equations. Appl. Math. Lett. 15, 575-583 (2002)

43. Wang, W, Sheng, J: Positive solutions to a multi-point boundary value problem with delay. Appl. Math. Comput. 188, 96-102 (2007)

44. Wang, Y, Zhao, W, Ge, W: Multiple positive solutions for boundary value problems of second order delay differential equations with one-dimensional p-Laplacian. J. Math. Anal. Appl. 326, 641-654 (2007)

45. Jankowski, T: Positive solutions for fourth-order differential equations with deviating arguments and integral boundary conditions. Nonlinear Anal. TMA 73, 1289-1299 (2010)

46. Jankowski, T: Multiple solutions of boundary-value problems for fourth-order differential equations with deviating arguments. J. Optim. Theory Appl. 146, 105-115 (2010) 
47. Guo, D, Lakshmikantham, V: Nonlinear Problems in Abstract Cones. Academic Press, New York (1988)

48. Feng, M: Existence of symmetric positive solutions for a boundary value problem with integral boundary conditions. Appl. Math. Lett. 24, 1419-1427 (2011)

49. Zhao, A, Yan, W, Yan, J: Existence of positive periodic solution for an impulsive delay differential equation. In: Topological Methods, Variational Methods and Their Applications, pp. 269-274. World Scientific, London (2002)

50. Wang, H: Positive periodic solutions of functional differential equations. J. Differ. Equ. 202, 354-366 (2004)

51. Sun, $\mathrm{H}, \mathrm{Li}, \mathrm{W}$ : Existence theory for positive solutions to one-dimensional $p$-Laplacian boundary value problems on time scales. J. Differ. Equ. 240, 217-248 (2007)

10.1186/1687-1847-2014-312

Cite this article as: Zhang and Feng: Transformation technique, fixed point theorem and positive solutions for second-order impulsive differential equations with deviating arguments. Advances in Difference Equations 2014, 2014:312

Submit your manuscript to a SpringerOpen ${ }^{\circ}$ journal and benefit from:

- Convenient online submission

- Rigorous peer review

- Immediate publication on acceptance

- Open access: articles freely available online

- High visibility within the field

- Retaining the copyright to your article

Submit your next manuscript at $>$ springeropen.com 